TRANSACTIONS OF THE

AMERICAN MATHEMATICAL SOCIETY

Volume 197, 1974

\title{
NONATTAINABILITY OF A SET BY A DIFFUSION PROCESS
}

BY

\section{A VNER FRIEDMAN( $\left.{ }^{1}\right)$}

ABSTRACT. Consider a system of $n$ stochastic differential equations $d \xi=b(\xi) d t+\sigma(\xi) d w$. Let $M$ be a $k$-dimensional submanifold in $R^{n}, k \leq$ $n-1$. For $x \in M$, denote by $d(x)$ the rank of $\sigma \sigma^{*}$ restricted to the linear space of all normals to $M$ at $x$. It is proved that if $d(x) \geq 2$ for all $x \in M$, then $\xi(t)$ does not hit $M$ at finite time, given $\xi(0) \xi M$, i.e., $M$ is nonattainable. The cases $d(x) \geq 1, d(x) \geq 0$ are also studied.

Introduction. It is well known that a Brownian motion in $n$-dimensions, $n \geq 2$, does not hit a prescribed point $x \neq 0$ with probability 1 . This result was recently extended by Bonami, Karoui, Roynette and Reinhard [1] to diffusion processes in $n$ dimension, $n \geq 2$, provided the diffusion matrix is nondegenerate. In another recent paper, Friedman and Pinsky [4] have proved that a diffusion process $\xi(t)$ does not hit a given closed domain $\Omega$, with probability 1 , provided $\xi(0) \notin \Omega$ and provided the "normal diffusion" and "normal drift" vanish on $\partial \Omega$.

The purpose of this paper is to prove general theorems of the form

$$
P_{x}\{\xi(t) \in M \text { for some } t>0\}=0 \text { if } x \notin M \text {, }
$$

where $\xi(t)$ is a diffusion process in $R^{n}$ and $M$ is a manifold in $R^{n}$ of dimension $k, 0 \leq k \leq n-1$. The result in [1] mentioned above will follow as a special case of one of the results (namely, The orem 4.1) of the present paper.

When $(0.1)$ holds we say that $M$ is nonattainable by the process $\xi(t)$.

Denote by $d(x)$ the rank of the diffusion matrix $a(x)$ at $x, x \in M$, when restricted to the linear subspace formed by the normals to $M$ at $x$. Our results depend in a crucial manner on $d(x)$.

In $\$ 1$ we give some basic definitions and prove a lemma, which is helpful in "localizing" the proof of nonattainability. In $\$ 2$ we reduce the problem of estab-

Received by the editors April 2, 1973. $35 \mathrm{~J} 25$.

AMS (MOS) subject classifications (1970). Primary 60H10; Secondary 60J60, 60J65,

Key words and phrases. Diffusion process, stochastic differential equations, Brownian motion, Dirichlet problem.

( $\left.{ }^{1}\right)$ This work was partially supported by National Science Grant GP-35347X. 
lishing (0.1) to the problem of finding a solution $u$ of $L u \leq \mu u$ near $M$, which "blows up" on $M ; L$ is the elliptic operator of the diffusion process and $\mu \geq 0$.

In $\$ 3$ we establish $(0.1)$ in the case $d \geq 3$. Under somewhat stronger assumptions we establish, in $\$ 4$, the property $(0.1)$ in case $d \geq 2$.

The cases $d=1$ and $d=0$ are dealt with in $\$ \$ 5$ and 6 respectively. Finally, in $\$ 7$, we consider the "mixed" case where $d=0$ on $M, d=1$ on $\partial M ; M$ is taken to be an arc in $R^{2}$. This case is motivated by applications to the Dirichlet problem for degenerate elliptic equations. Thus, it is shown in $\$ 7$ how recent results of Friedman and Pinsky [5] can be extended by using our. result on the "mixed" case.

Results of the type (0.1) when $n=1$ and $M$ consists of one point can be found in the book of Gikhman and Skorokhod [6] and in [1].

1. Basic definitions. A lemma. Let $M$ be a $k$-dimensional $C^{2}$ manifold in $R^{n}$. At each point $x^{0} \in M$, let $N^{k+i}\left(x^{0}\right)(1 \leq i \leq n-k)$ form a set of linearly independent vectors in $R^{n}$ which are normal to $M$ and $x^{0}$.

Let $a(x)$ be an $n \times n$ matrix, and consider the $(n-k) \times(n-k)$ matrix $\alpha=$ $\left(a_{i j}\right)$ where

$$
a_{i j}=\left\langle a\left(x^{0}\right) N^{k+i}\left(x^{0}\right), N^{k+j}\left(x^{0}\right)\right\rangle \quad(1 \leq i, j \leq n-k),
$$

here $\langle$,$\rangle denotes the scalar product in R^{n}$.

Denote the rank of $\alpha$ by $r_{M}{ }^{\perp}\left(x^{0}\right)$. This number is clearly independent of the choice of the particular set of normals $N^{k+i}\left(x^{0}\right)$.

Definition. The rank of $a(x)$ ortbogonal to $M$ at $x^{0}$ is the number $r_{M}{ }^{\perp}\left(x^{0}\right)$.

If the manifold $M$ has boundary $\partial M$, then we always take $M$ to be a closed set, i.e., $\bar{M}=M \cup \partial M=M$. If $x^{0} \in \partial M$, then by a normal $N$ to $M$ at $x^{0}$ we mean a vector $N$ that is $\lim N(x)$, where $x \in$ int $M, x \rightarrow x^{0}$ and $N(x)$ is normal to $M$ at $x$. We now define ${ }_{M}{ }_{M}\left(x^{0}\right)$, for $x^{0} \in \partial M$, in the same way as before.

Notice that ${ }^{M} \partial M$ is also a manifold, and one can define $r_{(\partial M)^{\perp}}\left(x^{0}\right)$. Clearly, $r_{(\partial M)^{\perp}}\left(x^{0}\right) \geq r_{M}^{\perp}\left(x^{0}\right)$.

Notice also that when $M$ consists of just one point $x^{0},{ }_{M^{\perp}}\left(x^{0}\right)$ is the rank of the matrix $a\left(x^{0}\right)$.

Consider now a diffusion process governed by a system of $n$ stochastic differential equations

$$
d \xi(t)=\sigma(\xi(t)) d w+b(\xi(t)) d t
$$

$\sigma(x)$ is an $n \times n$ matrix $\left(\sigma_{i j}(x)\right), b(x)$ is a column vector $\left(b_{1}(x), \cdots, b_{n}(x)\right)$, and $w(t)$ is an $n$ dimensional Brownian motion $\left(w^{1}(t), \cdots, w^{n}(t)\right)$.

We assume: 
(A)

$$
\sigma(x) \text { and } b(x) \text { satisfy, for all } x \in R^{n},
$$

$$
|\sigma(x)|+|b(x)| \leq C(1+|x|) \quad(C \text { constant })
$$

further, for any $R>0$ there is a positive constant $C_{R}$ such that $|\sigma(x)-\sigma(y)|+$ $|b(x)-b(y)| \leq C_{R}|x-y|$ if $|x|<R,|y|<R$.

Introduce the diffusion matrix $a(x)=\left(a_{i j}(x)\right)$ :

$$
a(x)=1 / 2 \sigma(x) \sigma^{*}(x) \quad\left[\sigma^{*}(x)=\text { transpose of } \sigma(x)\right],
$$

and denote the rank of $a(x)$ orthogonal to $M$ at $x$ by $d(x)$, i.e.,

$$
d(\dot{x})=r_{M^{\perp}}(x) \text { for } x \in M \text {. }
$$

Definition. A closed set $M$ in $R^{n}$ is nonattainable by the process $\xi(t)$ if

$$
P_{x}\{\xi(t) \in M \text { for some } t>0\}=0 \text { for each } x \notin M \text {. }
$$

It will be shown later on that if $d(x) \geq 2$ for all $x \in M$ (M a $C^{2}$ manifold) then $M$ is nonattainable. The same assertion is true in some cases when $d(x) \geq 1$ (but not always), provided $n \geq 2$. The interpretation of these results is that $M$ is "too thin" for $\xi(t)$ to hit it.

It will also be shown that when $d(x) \equiv 0$ on $M$, then the assertion (1.3) is still true provided the "normal drift" of $\xi(t)$ vanishes on $M$. The interpretation of this result is that $M$ is an "obstacle" for the diffusion process $\xi(t)$.

We conclude this section with a lemma that will be useful in reducing the proof of the assertion (1.3) from a global manifold $M$ to a local one.

Let $x^{0} \in M$. Then, in a neighborhood of $x^{0}, M$ can be represented in the form

$$
x_{i^{\prime}}=f_{i^{\prime}}\left(x^{\prime \prime}\right)
$$

where $i^{\prime}$ varies over $n-k$ of the indices $1,2, \cdots, n$, the coordinates of $x^{\prime \prime}$ are $x_{i \prime \prime}$, and $i^{\prime \prime}$ varies over the remaining indices. Suppose for simplicity that $i^{\prime}$ varies over $k+1, \cdots, n$, i.e., $M$ is given locally by

$$
x_{k+i}=f_{k+i}\left(x_{1}, \ldots, x_{k}\right) \quad(i=1, \ldots, n-k) \text {. }
$$

Introduce the mapping

$$
\begin{array}{rlrl}
y_{i} & =x_{i}-x_{i}^{0} & & (i=1, \ldots, k), \\
y_{k+i} & =x_{, k+i}-f_{k+i}\left(x_{1}, \ldots, x_{k}\right) & (i=1, \ldots, n-k),
\end{array}
$$

where $x^{0}=\left(x_{1}^{0}, \cdots, x_{n}^{0}\right)$. This is a diffeomorphism from a neighborhood $V\left(x^{0}\right)$ of $x^{0}$ into a neighborhood $V^{*}$ of 0 in the $y$-space. Denote by $M^{*}$ the image of $M \cap V\left(x^{0}\right)$. Then $M^{*}$ is given by 


$$
y_{i}=0 \quad(i=1, \ldots, k),\left(y_{k+1}, \ldots, y_{n}\right) \in A,
$$

for some set $A$.

Consider the elliptic operator

$$
L u=\sum_{i, j=1}^{n} a_{i j}(x) \frac{\partial^{2} u}{\partial x_{i} \partial x_{j}}+\sum_{i=1}^{n} b_{i}(x) \frac{\partial u}{\partial x_{i}}
$$

and set $v(y)=u(x)$. Then $L u(x)=L^{\prime} v(y)$ where

$$
L^{\prime} v=\sum_{i, j=1}^{n} a_{i j}^{*}(y) \frac{\partial^{2} v}{\partial y_{i} \partial y_{j}}+\sum_{i=1}^{n} b_{i}^{*}(y) \frac{\partial v}{\partial y_{i}} .
$$

It is easily seen that $a_{k+i, k+j}^{*}(y)=\left\langle a(x) N^{k+i}(x), N^{k+j}(x)\right\rangle$ where

$$
N^{k+i}(x)=\nabla_{x} g_{k+i}(x), \quad g_{k+i}(x)=x_{k+i}-f_{k+i}\left(x_{1}, \ldots, x_{k}\right) .
$$

Notice that if $x \in M \cap V\left(x^{0}\right)$ then the $N^{k+i}(x)(1 \leq i \leq n-k)$ form a set of linearly independent normal vectors to $M$ at $x$. Hence

$$
d(x)=\operatorname{rank}\left(a_{k+i, k+j}^{*}(x)\right)_{i, j=1}^{n-k} \quad\left(x \in M \cap V\left(x^{0}\right)\right) .
$$

By performing an affine transformation in the space of variables $\left(y_{k+1}, \cdots\right.$, $y_{n}$ ) we do not affect the manifold $M^{*}$ given by (1.7), except for a change in the set $A$. At the same time, after performing such a transformation we can achieve the conditions

$$
\hat{a}_{k+i, k+j}(0)=\left\{\begin{array}{l}
1 \quad \text { if } i=j=k+1, \ldots, d\left(x^{0}\right), \\
0 \quad \text { for all other } i, j(1 \leq i, j \leq n-k),
\end{array}\right.
$$

where $\hat{a}_{k+i, k+j}$ are the new $a_{k+i, k+j}^{*}$

Next, by an affine transformation in the space of variables $\left(y_{1}, \cdots, y_{k}\right)$ we do not affect the manifold $M^{*}$. At the same time we can achieve the additional conditions

$$
\tilde{a}_{i, j}(0)=\left\{\begin{array}{l}
\eta \quad \text { if } i=j=1, \ldots, d^{*}(\eta>0), \\
0 \text { for all other } i, j(1 \leq i, j \leq k),
\end{array}\right.
$$

where $\eta$ is any given positive number, $d^{*}$ is the rank of the matrix $\left(\hat{a}_{i j}(0)\right)_{i, j=1}^{k}$ and $\tilde{a}_{i, j}$ are the new $\hat{a}_{i, j}$. Notice that $d^{*}$ can be any number $\geq 0$ and $\leq k$.

Notation. Let $B$ be any set in $R^{n}$ and let $x \in R^{n}$. The distance from $x$ to $B$ will be denoted by $d(x, B)$. 
Let $M_{V}=M \cap V\left(x^{0}\right)$. Let $W$ be a neighborhood of $M_{V}$. We shall be interested, later on, in finding a function $u$ satisfying:

$$
\begin{aligned}
& L u(x) \leq \mu u(x) \text { if } x \in W \backslash M_{V} \quad(\mu \text { nonnegative constant }), \\
& u(x) \rightarrow \infty \text { if } x \in W \backslash M_{V}, d\left(x, M_{V}\right) \rightarrow 0 .
\end{aligned}
$$

Suppose after performing the transformation (1.6) and the two affine transformations used above (to get (1.9), (1.10)), we can construct a function $u^{\prime}\left(x^{\prime}\right)$ satisfying (1.11) in the new $x^{\prime}$-variable and with the transformed $L$ and $M$. Then the function $u(x)=u^{\prime}\left(x^{\prime}\right)$ will satisfy (1.11). Consequently, in trying to prove the existence of $u(x)$ satisfying (1.11), we may, without loss of generality, assume that $M$ is given by

$$
x_{k+1}=0, \ldots, x_{n}=0
$$

that $x^{0}=0$, and that

$$
a_{k+i, k+j}(0)=\left\{\begin{array}{l}
1 \quad \text { if } i=j=1, \ldots, d(0), \\
0 \quad \text { for all other } i, j ; 1 \leq i, j \leq n-k,
\end{array}\right.
$$

$$
a_{i, j}(0)=\left\{\begin{array}{l}
\eta \quad \text { if } i=j=1, \ldots, d^{*}(\eta>0), \\
0 \quad \text { for all other } i, j ; 1 \leq i, j \leq k,
\end{array}\right.
$$

for some $0 \leq d^{*} \leq k$.

In the above arguments we have assumed the local representation (1.5). The same arguments apply, of course, also in the general case where $M$ has a local representation of the form (1.4). We sum up:

Lemma 1.1. In order to find a function $u$ satisfying (1.11), we may assume, without loss of generality, that $x^{0}=0$, that $M$ is given by (1.12) and that (1.13), (1.14) bold.

The following result can be obtained by slightly modifying the proof of Lemma 1.1 .

Lemma 1.1'. Let $p$ be a given positive number. In order to find a function $u$ satisfying $L u(x) \leq-\mu /(d(x, M))^{p}$ if $x \in W \backslash M_{V}$ ( $\mu$ positive constant), $u(x) \rightarrow \infty$ if $x \in W \backslash M_{V}, d\left(x, \bar{M}_{V}\right) \rightarrow 0$, we may assume, without loss of generality, that $x^{0}=$ 0, that $M$ is given by (1.12) and that (1.13), (1.14) bold.

2. A fundamental lemma. A function $v(x)$ is said to be piecewise continuous in a region $G$ of $R^{n}$ if there is in $G$ a finite number of $C^{1}$ hypersurfaces $S_{1}, \cdots, S_{l}$ 
and a finite number of $C^{1}$ manifolds of dimensions $\leq n-2, V_{1}, \cdots, V_{b}$, such that:

(i) for any compact subset $G_{0}$ of $G, v(x)$ is continuous and bounded on the set $G_{0} \backslash(S \cup V)$ where $S=\bigcup_{i=1}^{l} S_{i}, V=\bigcup_{i=1}^{b} V_{i}$, and

(ii) $v(x)(x \in G \backslash(S \cup V))$ tends to a limit from either side of each $S_{i}$.

Notation. The gradient of $v$ is denoted by $D_{x} v$. The gradient of $D_{x} v$ is denoted by $D_{x}^{2} v$.

Let $\Omega$ be an open set in $R^{n}$. Denote by $\partial \Omega$ the boundary of $\Omega$, and by $\bar{\Omega}$ the closure of $\Omega$. Let

$$
r=\text { exit time of } \xi(t) \text { from } \Omega \text {. }
$$

Let $K$ be a compact subset of $\bar{\Omega}$. For any $\epsilon>0$, let

$$
K_{\epsilon}=\{x \in \Omega ; d(x, K) \leq \epsilon\}, \quad \hat{K}_{\epsilon}=K_{\epsilon} \backslash K .
$$

Notice that $K$ need not lie entirely in $\Omega$, i.e., $K \cap \partial \Omega$ may be nonempty. The following lemma will be fundamental for the subsequent developments.

Lemma 2.1. Let $(A)$ bold. Let $u$ be a continuously differentiable function in $\hat{K}_{\epsilon_{0}}$, for some $\epsilon_{0}>0$, and let $D_{x}^{2} u$ be piecewise continuous in $\hat{K}_{\epsilon_{0}}$. Denote by $s_{1}, \cdots, s_{l}$ the $(n-1)$-dimensional manifolds of discontinuity of $D_{x}^{2} u$, and by $V_{1}$, $\cdots, v_{b}$ the manifolds of discontinuity of $D_{x}^{2} u$ of dimensions $\leq n-2$. Let $S=$ $\bigcup_{i=1}^{l} s_{i}, V=\bigcup_{i=1}^{b} V_{i}$. Suppose

$$
\begin{gathered}
L u(x) \leq \mu u(x) \text { if } x \in \hat{K}_{\epsilon_{0}} \backslash(S \cup V)(\mu \text { positive constant }), \\
u(x) \rightarrow \infty \text { if } x \in \hat{K}_{\epsilon_{0}}, \quad d(x, K) \rightarrow 0 .
\end{gathered}
$$

Then, for any $x \in \Omega \backslash K$,

$$
P_{x}\{\xi(t) \in K \text { for some } 0 \leq t<\tau\}=0 \text {. }
$$

This lemma was implicitly stated and proved in [3], [4] in the special case where $K$ is a point or a bounded closed domain, $\Omega=R^{n}, \hat{K}_{\epsilon_{0}}$ is replaced by $R^{n}$, and $u$ is twice continuously differentiable in $R^{n} \backslash K$.

Proof. Let $R, \rho$ be positive numbers; $R$ will be arbitrarily large and $\rho$ arbitrarily small. Set 


$$
B_{R}=\{x ;|x|<R\}, \quad \Omega_{\rho}=\{x \in \Omega ; d(x, \partial \Omega)>\rho\} .
$$

$R$ is such that $K \subset B_{R}$.

Fix a number $\epsilon_{1}, 0<\epsilon_{1}<\epsilon_{0}$ and let $0<\epsilon^{\prime}<\epsilon<\epsilon_{1}$.

Modify and extend $u$ inside $K_{\epsilon}$ and outside $K_{\epsilon_{1}}$ so as to obtain a function $U$ in $\Omega$ satisfying:

$$
\begin{aligned}
& U \text { and } D_{x} U \text { are continuous in } \Omega \text {; } \\
& D_{x}^{2} U \text { is piecewise continuous in } \Omega \text {; }
\end{aligned}
$$

$U$ is positive in $\Omega$.

Since (by (2.2)) $u(x)$ is positive in some $\Omega$-neighborhood of $K$, we can accomplish (2.4) provided $\epsilon_{1}$ is sufficiently small.

Denote by $\Sigma$ the set of discontinuities of $D_{x}^{2} U$. Clearly, for any $\rho, R$,

$$
\begin{aligned}
\left|D_{x} U\right|+\left|D_{x}^{2} U\right| \leq C(\rho, R) & \text { if } x \in\left(\Omega_{\rho / 2} \backslash K_{\epsilon_{1}}\right) \cap B_{R+1}, x \notin \Sigma, \\
U \geq c(\rho, R) & \text { if } x \in\left(\Omega_{\rho / 2} \backslash K_{\epsilon_{1}}\right) \cap B_{R+1},
\end{aligned}
$$

where $C(\rho, R), c(\rho, R)$ are positive constants depending on $\rho, R$, but independent of $\epsilon^{\prime}$. Since $U=u$ in $K_{\epsilon_{1}} \backslash K_{\epsilon^{\prime}}$, we conclude, upon using (2.1) and (2.5), that

$$
L U(x) \leq \mu_{\rho, R} U(x) \text { if } x \in\left(\Omega_{\rho / 2} \backslash K_{\epsilon^{\prime}}\right) \cap B_{R+1}, x \notin \Sigma,
$$

where $\mu_{\rho, R}$ is a positive constant depending on $\rho, R$, but independent of $\epsilon^{\prime}$.

Let $p(x)$ be a $C^{\infty}$ function in $R^{n}$, with support in the unit ball $|x| \leq 1$, such that $p(x) \geq 0, \int_{R^{n}} p(x) d x=1$. For any $\lambda>0$, we introduce the mollifier $U_{\lambda}(x)$ of $U(x)$ defined by (cf. [2])

$$
U_{\lambda}(x)=\int_{|y-x|<\lambda} U(y) p_{\lambda}(x-y) d y \quad\left[p_{\lambda}(x)=\left(1 / \lambda^{n}\right) p(x / \lambda)\right]
$$

We take $\lambda<\rho / 2, \lambda<\epsilon-\epsilon^{\prime}, x \in \Omega_{\rho^{*}}$. Then $U_{\lambda}(x)$ is in $C^{\infty}\left(\Omega_{\rho}\right)$, and

$$
D_{x} U_{\lambda}(x)=\int_{|y-x|<\lambda} D_{y} U(y) \cdot p_{\lambda}(x-y) d y .
$$

Also,

$$
D_{x}^{2} U_{\lambda}(x)=-\int_{|y-x|<\lambda} D_{y} U(y) \cdot D_{y} p_{\lambda}(x-y) d y
$$

If $d(x, \Sigma)>\lambda$ then clearly 


$$
D_{x}^{2} U_{\lambda}(x)=\int_{|y-x|<\lambda} D_{y}^{2} U(y) \cdot p_{\lambda}(x-y) d y \text {. }
$$

Suppose next that $d(x, \Sigma) \leq \lambda$ and $\Sigma \cap\{y ;|y-x| \leq \lambda\}$ consists of a hypersurface $S_{1}$. Then $S_{1}$ divides $\{y ;|\bar{y}-x| \leq \lambda\}$ into two sets: $S_{1 \lambda}$ and $S_{2 \lambda}$. Integrating by parts in (2.9) over $S_{1 \lambda}$ and $S_{2 \lambda}$ separately, and using the continuity of $D_{y} U$ across $S_{1}$, we again get $(2.10)$.

If $\Sigma \cap\{y ;|y-x| \leq \lambda\}$ consists of manifold $V$ of dimension $\leq n-2$, then we surround $V$ by an $\eta$-neighborhood $V_{\eta}$, and split the integral in (2.9) into a part $I_{1}$ integrated over $\{y ;|y-x|<\lambda\} \cap V_{\eta}$ and a part $I_{2}$. In $I_{2}$ we integrate by parts so as to obtain

$$
l_{2}=\int_{W_{\eta}} D_{y}^{2} U(y) \cdot p_{\lambda}(x-y) d y+O(\eta), \quad W_{\eta}=\left\{y ;|y-x|<\lambda, y \notin V_{\eta}\right\}
$$

Taking $\eta \rightarrow 0$ in $I_{1}+I_{2},(2.10)$ follows.

Finally, the general case where $d(x, \Sigma) \leq \lambda$ can be handled by combining the above two special cases. Thus $(2.10)$ holds in general.

From (2.7), (2.8), and (2.10) we obtain

$$
L U_{\lambda}(x)-\mu_{\rho, R} U_{\lambda}(x)=\int_{|y-x|<\lambda}\left[L U(y)-\mu_{\rho, R} U(y)\right] p_{\lambda}(x-y) d y .
$$

Using (2.6), we get

$$
L U_{\lambda}(x) \leq \mu_{\rho, R} U_{\lambda}(x) \text { if } x \in\left(\Omega_{\rho} \backslash K_{\epsilon}\right) \cap B_{R}
$$

Let $\tau^{0}=\tau_{\rho, R, \epsilon}=$ exit time of $\xi(t)$ from $\left(\Omega_{\rho} \backslash K_{\epsilon}\right) \cap B_{R}$, and write, for simplicity, $\mu=\mu_{\rho, R}$. By Itô's formula, if $x \in\left(\Omega_{\rho} \backslash K_{\epsilon}\right) \cap B_{R}, T>0$, then

$$
\begin{aligned}
& E_{x}\left\{e^{-\mu\left(\tau^{0} \wedge T\right)} U_{\lambda}\left(\xi\left(\tau^{0} \wedge T\right)\right)\right\}-U_{\lambda}(x) \\
&=E_{x} \int_{0}^{\tau^{0} \wedge T} e^{-\mu_{s}}(L-\mu) U_{\lambda}(\xi(s)) d s
\end{aligned}
$$

Notice that $\xi(s) \epsilon\left(\Omega_{\rho} \backslash K_{\epsilon}\right) \cap B_{R}$ if $0 \leq s<\tau^{0} \wedge T$. Hence, by (2.11), the integral on the right-hand side is $\leq 0$. Taking $\lambda \rightarrow 0$ in (2.12) and using the fact that $U_{\lambda}(y) \rightarrow U(y)$ uniformly in $y \in\left(\Omega_{\rho} \backslash K_{\epsilon}\right) \cap B_{R}$, we get

$$
E_{x}\left\{e^{-\mu\left(\tau^{0} \wedge T\right)} U\left(\xi\left(\tau^{0} \wedge T\right)\right)\right\}-U(x) \leq 0 .
$$

Since $U>0$, this yields 


$$
E_{x}\left\{e^{-\mu\left(\tau^{0} \wedge T\right)} U\left(\xi\left(\tau^{0} \wedge T\right)\right) I\left\{\xi\left(\tau^{0} \wedge T\right) \epsilon \partial K_{\epsilon, \rho}\right\} \leq U(x)\right.
$$

where $\mu=\mu_{\rho, R}, \tau^{0}=\tau_{\rho, R, \epsilon}, \partial K_{\epsilon, \rho}=\partial K_{\epsilon} \cap \Omega_{\rho}$, and $\partial K_{\epsilon}$ is the boundary of $K_{\epsilon}$; here $I_{A}$ is the indicator function of a set $A$.

Noting that $U\left(\xi\left(\tau^{0} \wedge T\right)\right) \geq \inf _{\dot{\alpha} K_{\epsilon} \cap \Omega} u(y)$ if $\xi\left(\tau^{0} \wedge T\right) \epsilon \partial K_{\epsilon, \rho}$, and taking $T \rightarrow \infty$ in (2.13), we get

$$
E_{x}\left\{e^{-\mu \tau^{0}} I_{\left\{\tau^{0}<\infty\right\}}\left\{\xi\left(\tau^{0}\right) \in \partial K_{\epsilon, \rho}\right\} \leq U(x) /\left[\inf _{y \in \partial K_{\epsilon} \cap \Omega} u(y)\right] .\right.
$$

Suppose now that the assertion (2.3) is false. Then there exists a set $G$ of positive probability such that: if $\omega \in G$ then $\xi(t, \omega) \in K$ for some finite $t=$ $t^{*}(\omega)<\tau(\omega)$. This implies that for all small $\epsilon$, say $0<\epsilon<\epsilon^{*}, \xi(s, \omega) \epsilon \Omega_{\rho} \cap B_{R}$ if $0 \leq s \leq t_{\epsilon}$ for some small $\rho>0$ and large $R, \xi(s, \omega) \notin K_{\epsilon}$ if $0 \leq s<t_{\epsilon}$, and $\xi\left(t_{\epsilon}, \omega\right) \epsilon K_{\epsilon}$; here $t_{\epsilon}=t_{\epsilon}(\omega) \leq t^{*}(\omega), \rho$ and $R$ are independent of $\epsilon$ (but they depend on $\omega$ ) and one can take, for instance, $\epsilon^{*}=\epsilon_{1}$ where $\epsilon_{1}$ is as above.

Setting $\rho_{m}=1 / m, R_{m}=m$,

$$
G_{m}=G \cap\left\{r_{\rho_{m}, R_{m}, \epsilon}<\infty ; \xi\left(\tau_{\rho_{m}, R_{m}, \epsilon}\right) \in \partial K_{\epsilon, \rho_{m}} \text { for all } 0<\epsilon<\epsilon^{*}\right\}
$$

we then have: $G=\bigcup_{m=1}^{\infty} G_{m}$. Since $P_{x}(G)>0$, it follows that $P_{x}\left(G_{m}\right)>0$ for some $m$. If we take $\rho=\rho_{m}, R=R_{m}$ in (2.14), and let $\epsilon \rightarrow 0$, we obtain, after using (2.2),

$$
E_{x}\left\{\exp \left[-\mu_{\rho_{m}, R}{ }_{m}{ }^{\tau} \rho_{m}, R_{m}, \epsilon\right] \cdot I_{G_{m}}\right\} \rightarrow 0 \text { if } \epsilon \rightarrow 0
$$

This implies that for almost all $\omega \in G_{m}$,

$$
\tau_{\rho_{m}, R}, \epsilon(\omega) \rightarrow \infty \text { if } \epsilon \rightarrow 0 .
$$

But if $\omega \in G_{m}$ then ${ }^{\circ} \rho_{m}, R_{m}, \epsilon^{(\omega)} \leq t^{*}(\omega)<\infty$, which contradicts (2.15), since $P_{x}\left(G_{m}\right)>0$.

Remark. The above proof remains valid in case $u$ is continuous in $\hat{K}_{\epsilon_{0}}$ and has two strong derivatives in $L^{2}(A)$ for any compact subset $A$ of $\hat{K}_{\epsilon_{0}},(2.1)$ holds almost everywhere, and (2.2) holds. Indeed, the assertions (2.8), (2.10) are then valid by definition of strong derivatives (see [2]), and the rest of the proof is essentially the same.

3. The case $d(x) \geq 3$. When we speak of a manifold $M$ with boundary $\partial M$, it is always assumed that $M$ is a closed set, i.e., $\partial M \subset M$.

Theorem 3.1. Let $M$ be a k-dimensional $C^{2}$ submanifold of $R^{n}(0 \leq k \leq n-1)$ 
with $C^{2}$ boundary $\partial M$ ( $\partial M$ may be empty), and let $(A)$ bold. Suppose $d(x) \geq 3$ for each $x \in M$. Then (1.3) bolds, i.e., $M$ is nonattainable.

Proof. If the assertion is not true then for some $x \notin M$ there is a point $x^{0} \in M$ such that, for any $\delta_{0}>0$,

$$
P_{x}\left\{\xi(t) \in M \cap B_{\delta_{0}} \text { for some } t>0\right\}>0
$$

where $B_{\delta_{0}}$ is the closed ball with center $x^{0}$ and radius $\delta_{0}$.

Consider first the case where $x^{0} \notin \partial M$. We want to apply Lemma 2.1 with $\Omega=R^{n}, K=M \cap B_{\delta_{0}}$. Thus we wish to construct a function $u$ in a $\delta$-neighborhood $W_{\delta}$ of $K$ such that

$$
\begin{array}{ll}
L u(x) \leq \mu u(x) & \text { if } x \in W_{\delta} \backslash K(\mu \geq 0), \\
u(x) \rightarrow \infty & \text { if } x \in W_{\delta} \backslash K, d(x, K) \rightarrow 0 .
\end{array}
$$

In view of Lemma 1.1, we may assume that $x^{0}=0$,

$$
K=\left\{x ; x_{k+1}=0, \ldots, x_{n}=0,\left(x_{1}, \ldots, x_{k}\right) \in A\right\}
$$

and that the $a_{i j}(x)$ satisfy (1.13), (1.14) with a given arbitrarily small $\eta>0$. Further, since $\delta_{0}$ can be taken arbitrarily small, we may assume that $A$ is a $k$ dimensional cube, say

$$
A=A_{\epsilon}=\left\{\left(x_{1}, \ldots, x_{k}\right) ;-\epsilon \leq x_{i} \leq \epsilon \text { for } i=1, \ldots, k\right\}
$$

and $\epsilon$ is sufficiently small. We shall determine later on how small $\epsilon$ and $\eta$ are going to be. Also $\delta$ can be taken arbitrarily small.

Set $x=\left(x^{\prime}, x^{\prime \prime}\right)$ where $x^{\prime}=\left(x_{1}, \cdots, x_{k}\right), x^{\prime \prime}=\left(x_{k+1}, \cdots, x_{n}\right)$, and let $r=$ $r(x)=\left|x^{\prime \prime}\right|$. Thus $r(x)$ is the distance from $x$ to $K$ provided $x^{\prime} \in A_{\epsilon}$.

Let

$$
u(x)=\phi(r)=\log r^{-1} \quad \text { if } x \in W_{\delta} \backslash K, x^{\prime} \in A_{\dot{\epsilon}} \cdot
$$

Then $u_{x_{i}}=-x_{i} / r^{2}, u_{x_{i} x_{j}}=-\delta_{i j} / r^{2}+2\left(x_{i} x_{j} / r^{2}\right)$ if $k+1 \leq i, j \leq n$, and $u_{x_{i} x_{j}}=$ 0 otherwise. Hence, if $d=d(0)$,

$$
\sum_{i=1}^{d} a_{k+i, k+i}(0) \frac{\partial^{2} u}{\partial x_{k+i}^{2}}=-\frac{d}{r^{2}}+2 \frac{x_{k+1}^{2}+\cdots+x_{k+d}^{2}}{r^{4}} \leq-\frac{1}{r^{2}}
$$

since $d \geq 3$. If $i=j>d$ or if $i \neq j, k+1 \leq i, j \leq n$, then 


$$
\begin{aligned}
\left|a_{k+i, k+j}(x) \frac{\partial^{2} u}{\partial x_{k+i} \partial x_{k+j}}\right| & =\left|a_{k+i, k+j}(x)-a_{k+i, k+j}(0)\right|\left|\frac{\partial^{2} u}{\partial x_{k+i} \partial x_{k+j}}\right| \\
& \leq C|x| / r^{2} \leq C(\delta+\epsilon) / r^{2}
\end{aligned}
$$

where $C$ is a generic constant. Also

$$
\left|\left[a_{k+i, k+i}(x)-a_{k+i, k+i}(0)\right] \frac{\partial^{2} u}{\partial x_{k+i}^{2}}\right| \leq \frac{C(\delta+\epsilon)}{r^{2}} \text { if } 1 \leq i \leq d .
$$

Noting also that $a_{i j} u_{x_{i} x_{j}}=0$ if either $1 \leq i \leq k$ or $1 \leq j \leq k$, and that $\left|b_{i} u_{x_{i}}\right| \leq$ $C\left|u_{x_{i}}\right| \leq C / r$, we conclude that

$$
L u \leq-\frac{1}{r^{2}}+\frac{C(\delta+\epsilon)}{r^{2}}<-\frac{1}{2 r^{2}} \quad \text { if } x \in W_{\delta} \backslash K, x^{\prime} \in A_{\epsilon}
$$

provided $\delta+\epsilon<1 /(2 C)$.

We next extend the definition of $u(x)$ to the set of points $\left(x^{\prime}, x^{\prime \prime}\right)$ in $W_{\delta} \backslash K$ where $x^{\prime} \notin A_{\epsilon}$. We begin with the subset where

$$
x_{1}>\epsilon, \quad-\epsilon \leq x_{i} \leq \epsilon \text { if } 2 \leq i \leq k
$$

Let $r_{1}=r_{1}(x)=\left\{\left(x_{1}-\epsilon\right)^{2}+\left|x^{\prime \prime}\right|^{2}\right\}^{1 / 2}$ if $x \in W_{\delta} \backslash K, x^{\prime}$ satisfies (3.6). Thus $r_{1}(x)$ is the distance from $x$ to $K$. Define $u(x)=\log 1 / r_{1}$ if $x \in W_{\delta} \backslash K$ and $x^{\prime}$ satisfies (3.6).

Denote by $L^{\prime}$ the operator $L$ when $a_{11}(x)$ and the $a_{1, k+i}(x), a_{k+i, 1}(x)$ $(1 \leq i \leq d)$ are replaced by 0 . Then, by the same calculation as before,

$$
L^{\prime} u(x)<-1 / 2 r_{1}^{2}
$$

Since $a_{11}(0)=\eta, a_{11}(x)<\eta+C(\delta+\epsilon)$ if $x \in W_{\delta}$. Recalling that $a(x)$ is a positive semidefinite matrix, we also have

$$
\left|a_{1, k+i}(x)\right| \leq \sqrt{a_{11}(x)} \sqrt{a_{k+i, k+i}(x)} \leq C(\eta+\delta+\epsilon)^{1 / 2}\left(x \in W_{\delta}\right) .
$$

Since $\left|\partial^{2} u / \partial x_{1} \partial x_{j}\right| \leq 3 / r_{1}^{2}$, we conclude that $\left|L u-L^{\prime} u\right| \leq 6 n C(\eta+\varepsilon+\epsilon)^{1 / 2} / r_{1}^{2}$ $\left(x \in W_{\delta}, x^{\prime}\right.$ satisfies (3.6)). Combining this with (3.7) and taking $\eta$ (and $\delta, \epsilon$ ) to be sufficiently small, we get $L u(x)<-1 / 3 r_{1}^{2}$ if $x \in W_{\delta} \backslash K, x^{\prime}$ satisfies (3.6).

Notice that $r^{2}$ and $r_{1}^{2}$ agree with the ir first derivatives on the set where $x_{1}=\epsilon_{0}$. Hence the function $u(x)$ constructed so far is continuously differentiable, and $D_{x}^{2} u$ is piecewise continuous.

Similarly we extend the definition of $u(x)$ to each of the subsets $M_{i}, N_{i}$ $(1 \leq i \leq k)$ of $W_{\delta} \backslash K$ given by 


$$
\begin{aligned}
& M_{i}=\left\{x \in W_{\delta} \backslash K, x_{i}>\epsilon,-\epsilon \leq x_{j} \leq \epsilon \text { if } 1 \leq j \leq k, j \neq i\right\}, \\
& N_{i}=\left\{x \in W_{\delta} \backslash K, x_{i}<-\epsilon,-\epsilon \leq x_{j} \leq \epsilon \text { if } 1 \leq j \leq k, j \neq i\right\} .
\end{aligned}
$$

Next we extend the definition of $u(x)$ to the subset $\Gamma$ of $W_{\delta} \backslash K$ where $x_{1}>\epsilon$, $x_{2}>\epsilon_{\text {. Introducing }}$

$$
r_{12}(x)=\left\{\left(x_{1}-\epsilon\right)^{2}+\left(x_{2}-\epsilon\right)^{2}+\left|x^{\prime \prime}\right|^{2}\right\}^{1 / 2},
$$

we define $u(x)=\log \left(1 / r_{12}(x)\right)$. Again we have (if $\eta, \delta, \epsilon$ are sufficiently small) $L u<-c /\left(r_{12}\right)^{2}$ for some positive constant $c$. Notice that the functions $r_{12}^{2}, r_{1}^{2}$ and their first derivatives agree on the set $x_{2}=\epsilon$. Similarly the functions $r_{12}^{2}$ and $r_{2}^{2}=\left(x_{2}-\epsilon\right)^{2}+\left|x^{\prime \prime}\right|^{2}$ and the ir first derivatives agree on the set $x_{1}=\epsilon$. Hence, the function $u(x)$ constructed so far is continuously differentiable, and $D_{x}^{2} u$ is piecewise continuous.

We extend the definition of $u$, in a similar manner, to the subsets of $w_{\delta} \backslash K$ defined by $x_{i}>\epsilon, x_{j}>\epsilon$, or $x_{i}>\epsilon, x_{j}<-\epsilon$, or $x_{i}<-\epsilon, x_{j}<-\epsilon$, for some $i \neq j, 1 \leq i, j \leq k$. Then we proceed to define $u(x)$ on sets determined by three inequalities, i.e., $x_{i}>\epsilon$ or $x_{i}<-\epsilon, x_{j}>\epsilon$ or $x_{j}<-\epsilon, x_{h}>\epsilon$ or $x_{h}<-\epsilon$; etc. The resulting function $u(x)$ is continuously differentiable in the entire set $W_{\delta} \backslash K$, $D_{x}^{2} u$ is piecewise continuous, and $L u(x)<0$ at all the points of $W_{\delta} \backslash K$ where $D_{x}^{2} u$ exists. Finally, it is clear that $u(x) \rightarrow \infty$ if $x \in W_{\delta} \backslash K, d(x, K) \rightarrow 0$.

Having constructed $u$ which satisfies (3.2) in the special case where (3.3) and (1.13), (1.14) hold, we appeal to Lemma 1.1 in order to conclude the existence of a continuously differentiable function $u$, with $D_{x}^{2} u$ piecew ise continuous, which satisfies (3.2) in the general case where $K=M \cap B_{\delta_{0}}$. Applying Lemma 2.1, it follows that

$$
P_{x}\{\xi(t) \in K \text { for some } t>0\}=0 \text { for any } x \notin K \text {. }
$$

This, however, contradicts (3.1).

We have assumed so far that $x^{0} \notin \partial M$. If $x^{0} \in \partial M$ then the proof is similar. The set $A_{\epsilon}$ is simply replaced by its intersection with the half space $x_{1} \geq 0$.

4. The case $d(x) \geq 2$. We first consider the case where $M$ consists of one point $x^{0}$. The number $d\left(x^{0}\right)$ now means the rank of the matrix $a\left(x^{0}\right)$.

Theorem 4.1. Let $(\mathrm{A})$ bold and let $d\left(x^{0}\right) \geq 2$. Then

$$
P_{x}\left\{\xi(t)=x^{0} \text { for some } t>0\right\}=0 \text { for any } x \neq x^{0} \text {. }
$$

Proof. We may take $x^{0}=0$. We wish to construct a function $u$ such that

$$
L u(x) \leq 0 \quad \text { if } 0<|x|<\delta,
$$




$$
u(x) \rightarrow \infty \text { if }|x| \rightarrow 0,
$$

where $\delta$ is a sufficiently small positive number, and $u(x)$ is in $C^{2}$ for $0<|x|<\delta$. In view of Lemma 2.1, this will complete the proof of (4.1).

Because of Lemma 1.1, we may assume, without loss of generality, that

$$
\begin{array}{ll}
a_{i i}(0)=1 & \text { if } i=1, \ldots, d, \\
a_{i j}(0)=0 & \text { if } i=j>d \text { or if } i \neq j .
\end{array}
$$

We shall take $u(x)=\phi(r)$ where $r=|x|$ and where $\phi(r)$ is defined by

$$
\phi^{\prime}(r)=-e^{r^{\theta} / \theta} / r, \quad \phi(0)=\infty
$$

for some constant $\theta, 0<\theta<1$. Since (4.3) clearly holds, it remains to verify (4.2). Now,

$$
u_{x_{i}}=-\frac{x_{i}}{r^{2}} e^{r} / \theta, \quad u_{x_{i} x_{j}}=\left[-\frac{\delta_{i j}}{r^{2}}+2 \frac{x_{i} x_{j}}{r^{4}}-\frac{x_{i} x_{j}}{r^{4}} r^{\theta}\right] e^{r / \theta} .
$$

Using the fact that $d \geq 2$, we get

$$
\begin{aligned}
\sum_{i=1}^{d} \frac{\partial^{2} u}{\partial x_{i}^{2}} & =\left[-\frac{d}{r^{2}}+2 \frac{x_{1}^{2}+\cdots+x_{d}^{2}}{r^{4}}-\frac{x_{1}^{2}+\cdots+x_{d}^{2}}{r^{4}} r^{\theta}\right] e^{r / \theta} \\
& \leq\left[-2 \frac{x_{d+1}^{2}+\cdots+x_{n}^{2}}{r^{4}}-\frac{x_{1}^{2}+\cdots+x_{d}^{2}}{r^{4}} r^{\theta}\right] e^{r / \theta} \leq-\frac{r^{\theta}}{r^{2}}
\end{aligned}
$$

if $r<1$. On the other hand,

$$
\left|\left[a_{i j}(x)-a_{i j}(0)\right] u_{x_{i} x_{j}}\right| \leq C|x| \frac{1}{r^{2}} \leq \frac{C}{r}, \quad\left|b_{i}(x) u_{x_{i}}\right| \leq C\left|u_{x_{i}}\right| \leq \frac{C}{r} .
$$

Recalling (4.4), we conclude that $L u \leq-r^{\theta} / r^{2}+C / r<0$ if $0<r<\delta$ and $\delta$ is sufficiently small. This completes the proof of $(4.2)$ and thereby also the proof of Theorem 4.1.

We shall now consider the case of a general manifold $M$ (without boundary). By Lemma 1.1, for any $x^{0} \in M$ there is a suitable diffeomorphism of a neighborhood $W$ of $x^{0}$ such that in the new coordinates $W \cap M$ has the form

$$
x_{k+1}=0, \cdots, x_{n}=0, \quad x_{1}^{2}+\cdots+x_{k}^{2} \leq \delta^{2}\left(x^{0}=0\right)
$$

and $a(x)$ satisfies (1.13), (1.14). Set $x=\left(x^{\prime}, x^{\prime \prime}\right), x^{\prime}=\left(x_{1}, \cdots, x_{k}\right), x^{\prime \prime}=$ $\left(x_{k+1}, \cdots, x_{n}\right), a_{\lambda \mu}\left(x^{\prime}\right)=a_{k+\lambda, k+\mu}\left(x^{\prime}, 0\right)(1 \leq \lambda, \mu \leq n-k)$. 
Denote by $a\left(x^{\prime}\right)$ the $(n-k) \times(n-k)$ matrix $\left(\alpha_{i j}\left(x^{\prime}\right)\right)$. If $d\left(x^{0}\right)=2$ and $n-k>2$, we introduce the $(n-k) \times(n-k)$ symmetric matrix $\alpha_{\epsilon}^{0}\left(x^{\prime}\right)=\left(\alpha_{i j}^{0}\left(x^{\prime}\right)\right)$ $(\epsilon>0)$, where

$$
\begin{aligned}
& \alpha_{11}^{0}\left(x^{\prime}\right)=\alpha_{22}^{0}\left(x^{\prime}\right)=(1-\epsilon) \sum_{\lambda=3}^{n-k} \alpha_{\lambda \lambda}\left(x^{\prime}\right), \quad \alpha_{12}^{0}\left(x^{\prime}\right)=0, \\
& a_{1 j}^{0}\left(x^{\prime}\right)=-2 \alpha_{1 j}\left(x^{\prime}\right), \quad \alpha_{2 j}^{0}\left(x^{\prime}\right)=-2 \alpha_{2 j}\left(x^{\prime}\right)(3 \leq j \leq n-k), \\
& \alpha_{i i}\left(x^{\prime}\right)=2-\epsilon(3 \leq i \leq n-k), \quad \alpha_{i j}\left(x^{\prime}\right)=0(3 \leq i \leq j \leq n-k, i \neq j) .
\end{aligned}
$$

We shall require the condition:

If $d\left(x^{0}\right)=2$ and $n-k>2$, then, for some $\epsilon>0$, $\left(\mathrm{N}_{x}{ }_{0}\right) \quad$ the matrix $a_{\epsilon}^{0}\left(x^{\prime}\right)$ is positive semidefinite for all $\left|x^{\prime}\right|$ sufficiently, small.

Definition. Let $n-k>2$. If at each point $x^{0} \in M$ where $d\left(x^{0}\right)=2$ the condition $\left(\mathrm{N}_{x}{ }_{0}\right)$ holds, then we say that the condition $(\mathrm{N})$ is satisfied.

Recall that $\alpha\left(x^{\prime}\right)$ is positive semidefinite. Hence $a_{i j}^{2} \leq \alpha_{i i} \alpha_{j j}$. It follows that, for any $\epsilon^{\prime}>0,\left|a_{i j}\left(x^{\prime}\right)\right| \leq\left(1+\epsilon^{\prime}\right) \sqrt{a_{j j}\left(x^{\prime}\right)}$ if $1 \leq i \leq 2,3 \leq j \leq n$. It is easily seen that if, for some $0<\theta<1 / 2,\left|\alpha_{i j}\left(x^{\prime}\right)\right| \leq \theta \sqrt{\alpha_{j j}\left(x^{\prime}\right)}$ if $1 \leq i \leq 2,3 \leq j \leq n$, for all $\left|x^{\prime}\right|$ sufficiently small, then the matrix $a_{\epsilon}^{0}\left(x^{\prime}\right)$ is positive definite, for some $\epsilon>0$, provided $\left|x^{\prime}\right|$ is sufficiently small; hence $\left(\mathrm{N}_{x} 0\right)$ follows in this case.

If $n-k=3$, the positivity of $\alpha\left(x^{\prime}\right)$ implies, for any $\epsilon^{\prime}>0$, that $\alpha_{13}^{2}\left(x^{\prime}\right)+$ $a_{23}^{2}\left(x^{\prime}\right) \leq\left(1+\epsilon^{\prime}\right) a_{33}\left(x^{\prime}\right)$ provided $\left|x^{\prime}\right|$ is sufficiently small. If $\alpha_{13}^{2}\left(x^{\prime}\right)+$ $a_{23}^{2}\left(x^{\prime}\right) \leq\left(1 / 2-\epsilon_{0}\right) a_{33}\left(x^{\prime}\right)$ for some $\epsilon_{0}>0$, then $\alpha_{\epsilon}^{0}\left(x^{\prime}\right)$ is positive definite for some $\epsilon>0$, provided $\left|x^{\prime}\right|$ is sufficiently small; hence $\left(\mathrm{N}_{x_{0}}\right)$ follows in this case.

The orem 4.2. Let $M$ be a $k$-dimensional $C^{2}$ submanifold of $R^{n}(0 \leq k \leq n-1)$, and let $(A)$ bold. Assume also that $a(x)$ is twice continuously differentiable in a neighborbood of $M$. If $d(x) \geq 2$ and if either $n-k=2$ or $(\mathrm{N})$ bolds, then (1.3) is satisfied, i.e., $M$ is nonattainable.

Proof. Consider first the case where $M$ is bounded. Let $x^{0} \in M$ and let $B_{\delta}$ be a closed ball with center $x^{0}$ and radius $\delta$. We wish to construct a function $u$ in $B_{\delta} \backslash M$ such that

$$
\begin{array}{rlrl}
L u(x) & \leq-c(d(x, M))^{\theta-2} & & \text { if } x \in B_{\delta} \backslash M(c>0,0<\theta<1), \\
\left|D_{x} u(x)\right| \leq C / d(x, M) & & \text { if } x \in B_{\delta} \backslash M, \\
u(x) & \rightarrow \infty & \text { if } x \in B_{\delta} \backslash M, d(x, M) \rightarrow 0 .
\end{array}
$$


We first consider the case where $x^{0}=0, B_{\delta} \cap M$ is given by (4.6), and (when $n-k \geq 3$ ) ( $\mathrm{N}_{x}$ ) holds. If $d=d(0) \geq 3$ then we can construct $u$ as in the proof of Theorem 3.1 (even with $\theta=0$ ). We shall therefore consider only the case $d=2$.

Let $m=n-k, x^{\prime \prime}=\left(x_{k+1}, \cdots, x_{n}\right)=\left(y_{1}, \cdots, y_{m}\right)$ and introduce the distance function

$$
r(x)=\left\{\sum_{i, j=1}^{m} b_{i j}\left(x^{\prime}\right) y_{i} y_{j}\right\}^{1 / 2}, \quad b_{i j}\left(x^{\prime}\right)=b_{j i}\left(x^{\prime}\right),
$$

where the $b_{i j}\left(x^{\prime}\right)$ are still to be determined, and $b_{i j}(0)=\delta_{i j}$. Let $\phi(r)$ be the function defined by (4.5). We wish to determine the $b_{i j}\left(x^{\prime}\right)$ in such a way that the function $u(x)=\phi(r(x))$ satisfies (4.7)-(4.9), provided $\delta$ is sufficiently small.

Clearly,

$$
\begin{aligned}
& \frac{\partial u}{\partial y_{\lambda}}=-\frac{1}{r^{2}}\left(\sum_{i=1}^{m} b_{i \lambda} y_{i}\right) e^{r / \theta}, \\
& \frac{\partial^{2} u}{\partial y_{\lambda} \partial y_{\mu}}=\left[-\frac{1}{r^{2}} b_{\lambda \mu}+\frac{2}{r^{4}}\left(\sum_{i=1}^{m} b_{i \lambda} y_{i}\right)\left(\sum_{j=1}^{m} b_{j \mu} y_{j}\right)\right. \\
&\left.-\frac{r^{\theta}}{r^{4}}\left(\sum_{i=1}^{m} b_{i \lambda} y_{i}\right)\left(\sum_{j=1}^{m} b_{j \mu} y_{j}\right)\right] e^{r / \theta} .
\end{aligned}
$$

Hence,

$$
\begin{array}{r}
\sum_{\lambda, \mu=1}^{m} a_{\lambda \mu} \frac{\partial^{2} u}{\partial x_{\lambda} \partial x_{\mu}}=\left[-\frac{1}{r^{2}} \sum_{\lambda, \mu=1}^{m} a_{\lambda \mu} b_{\lambda \mu}+\frac{2}{r^{4}} \sum_{\lambda, \mu=1}^{m} a_{\lambda \mu}\left(\sum_{i=1}^{m} b_{i \lambda} y_{i}\right)\left(\sum_{j=1}^{m} b_{j \mu} y_{j}\right)\right. \\
\left.-\frac{r^{\theta}}{r^{2}} \sum_{\lambda, \mu=1}^{m} a_{\lambda \mu}\left(\sum_{i=1}^{m} b_{i \lambda} y_{i}\right)\left(\sum_{j=1}^{m} b_{j \mu} y_{j}\right)\right] e^{r / \theta} .
\end{array}
$$

One is tempted to solve the system

$$
F_{i j} \equiv b_{i j} \sum_{\lambda, \mu=1}^{m} a_{\lambda \mu} b_{\lambda \mu}-2 \sum_{\lambda, \mu=1}^{m} a_{\lambda \mu} b_{i \lambda} b_{j \mu}=2\left(\alpha_{i j}(0)-\delta_{i j}\right)
$$

in a neighborhood of $x^{\prime}=0, b_{i j}=\delta_{i j}$, in the form $b_{i j}=b_{i j}\left(x^{\prime}\right)$. Unfortunately, the Jacobian vanishes at the point where $x^{\prime}=0, b_{i j}=\delta_{i j}$. We therefore proceed differently. We define $b_{11}=a_{22}, b_{22}=a_{11}, b_{12}=-a_{12}, b_{j j}=1$ if $3 \leq j \leq m$, $b_{i j}=0$ if $1 \leq i \leq j, j \geq 3, i \neq j$. Set $A=\sum_{\lambda=3}^{m} a_{\lambda \lambda}$, in case $m \geq 3$. One can easily check that $F_{i j}=0$ if $m=2$ and $1 \leq i \leq j \leq 2$. If $m>2$, then 


$$
\begin{aligned}
& F_{11}=a_{22} A, \quad F_{22}=a_{11} A, \quad F_{12}=-a_{12} A, \\
& F_{1 j}=-2 a_{22} a_{i j}+2 a_{12} a_{2 j}, \quad F_{2 j}=-2 a_{11} a_{2 j}+2 a_{12} a_{1 j} \quad(3 \leq j \leq m), \\
& F_{j j}=\sum_{\lambda=1}^{m} a_{\lambda \lambda} b_{\lambda \lambda}-2 a_{i j}=2+O\left(\left|x^{\prime}\right|\right) \quad \text { if } 3 \leq j \leq m, \\
& F_{i j}=-2 a_{i j} \text { if } 3 \leq i \leq j \leq m, i \neq j .
\end{aligned}
$$

Suppose $m \geq 3$. Using the condition $\left(\mathrm{N}_{x} 0\right)$ we find that

$$
\sum_{i, j=1}^{m} F_{i j} y_{i} y_{i} \geq \theta_{0}\left(y_{3}^{2}+\cdots+y_{m}^{2}\right) \text { for some } \theta_{0}>0
$$

provided $\delta$ is sufficiently small. Using this in (4.10), and noting that

$$
-\sum_{\lambda, \mu=1}^{m} a_{\lambda \mu}\left(\sum_{i=1}^{m} b_{i \lambda} y_{i}\right)\left(\sum_{j=1}^{m} b_{j \mu} y_{j}\right)=-y_{1}^{2}-y_{2}^{2}+\sum_{i, j=1}^{m} O\left(\left|x^{\prime}\right|\right) y_{i} y_{j},
$$

we get

$$
\begin{gathered}
\sum_{\lambda, \mu=1}^{m} a_{\lambda \mu}\left(x^{\prime}\right) \frac{\partial^{2} u}{\partial x_{\lambda} \partial x_{\mu}} \leq\left[-\theta_{0} \frac{y_{3}^{2}+\cdots+y_{m}^{2}}{r^{4}}-r \frac{\theta_{1}^{y_{1}^{2}+y_{2}^{2}}}{r^{4}}+O\left(\left|x^{\prime}\right|\right) \frac{r^{\theta}}{r^{2}}\right] e^{r^{\theta} / \theta} \\
\leq\left[-\theta_{0} \frac{r^{\theta}|y|^{2}}{r^{4}}+O\left(\left|x^{\prime}\right|\right) \frac{r^{\theta}}{r^{2}}\right] e^{r^{\theta} / \theta} \leq-\frac{1}{2} \theta_{0} \frac{r^{\theta}}{r^{2}}
\end{gathered}
$$

provided $\delta$ is sufficiently small. The final inequality is valid (by obvious modifications in the proof) also when $m=2$.

Next, if $1 \leq l, b \leq k, 1 \leq i \leq m$,

$$
\frac{\partial r}{\partial x_{l}}=O(r), \quad \frac{\partial^{2} r}{\partial x_{l} \partial x_{b}}=O(r), \quad \frac{\partial r}{\partial x_{k+i}}=O(1), \quad \frac{\partial^{2} r}{\partial x_{l} \partial x_{k+i}}=O(1) .
$$

Hence,

$$
\frac{\partial u}{\partial x_{l}}=O(1), \quad \frac{\partial^{2} u}{\partial x_{l} \partial x_{b}}=O(1), \quad \frac{\partial u}{\partial x_{k+i}}=O\left(\frac{1}{r}\right), \quad \frac{\partial^{2} u}{\partial x_{l} \partial x_{k+i}}=O\left(\frac{1}{r}\right) .
$$

Further,

$$
\left|\left[a_{k+\lambda, k+\mu}\left(x^{\prime}, x^{\prime \prime}\right)-a_{k+\lambda, k+\mu}\left(x^{\prime}, 0\right)\right] \frac{\partial^{2} u}{\partial x_{k+\lambda} \partial x_{k+\mu}}\right| \leq C\left|x^{\prime \prime}\right| \frac{C}{r^{2}}=\frac{C}{r} .
$$

From (4.11) and the subsequent estimates it follows that 


$$
L u \leq-1 / 2 \theta_{0} r^{\theta} / r^{2}+C / r \leq-c r^{\theta} / r^{2} \quad(c>0)
$$

provided $\delta$ is sufficiently small. Thus (4.7) has been established. The assertions (4.8), (4.9) obviously hold.

Having established (4.7)-(4.9) in the special coordinates where $B_{\delta} \cap M$ is given by (4.6) and (1.13) holds, we can now return to the original coordinates, and conclude (cf. Lemma 1.1'):

For every $y \in M$ there is a ball $B\left(y, \delta_{y}\right)$ with center $y$ and radius $\delta_{y}$ and a $C^{2}$ function $u^{y}(x)$ defined in $B\left(y, \delta_{y}\right) \backslash M$, such that

$$
L u^{y}<-c(d(x, M))^{\theta-2} \text { if } x \in B\left(y, \delta_{y}\right) \backslash M(c>0),
$$

$$
\left|D_{x} u^{y}(x)\right| \leq C / d(x, M) \text { if } x \in B\left(y, \delta_{y}\right) \backslash M
$$

$$
u^{y}(x) \rightarrow \infty \quad \text { if } x \in B\left(y, \delta_{y}\right) \backslash M, d(x, M) \rightarrow 0
$$

Cover a small neighborhood $W$ of $M$ by a finite number of balls $B\left(y, \delta_{y}\right)$. Denote these balls by $B_{i}=B\left(y_{i}, \delta_{y_{i}}\right)$ and the corresponding functions $u^{y}(x)$ by $u^{i}(x) ; 1 \leq i \leq l$.

Let $\left\{\zeta_{i}\right\}$ be a partition of unity subordinate to the covering $\left\{B_{i}\right\}$, and set

$$
u_{i}(x)= \begin{cases}\zeta_{i} u^{i} & \text { if } x \in B_{i} \backslash M \\ 0 & \text { if } x \notin B_{i}\end{cases}
$$

Since $\zeta_{i}=0$ outside $B_{i}, u_{i}(x)$ is in $C^{2}(W \backslash M)$. Further, by (4.12), (4.13),

$$
L u_{i} \leq \zeta_{i} L u^{i}+C / d(x, M) \leq-c \zeta_{i}(d(x, M))^{\theta-2}+C / d(x, M)
$$

if $x \in B_{i} \backslash M$. Setting $u=\sum_{i=1}^{l} u_{i}$, we get

$$
L u \leq-c \sum_{i=1}^{l} \zeta_{i}(d(x, M))^{2-\theta}+\frac{C}{d(x, M)}<0
$$

if $x \in W \backslash M$ and $c(d(x, M))^{1-\theta}<1 / C$, since $\Sigma \zeta_{i}=1$ on $W$.

From (4.14) we also have $u(x)=\sum_{i=1}^{l} \zeta_{i}(x) u^{i}(x) \rightarrow \infty$ if $x \in W \backslash M, d(x, M) \rightarrow$ 0 . An application of Lemma 2.1 with $\Omega=R^{n}, K=M$ now yields the assertion of Theorem 4.2, in case $M$ is a bounded set.

Consider next the case where the set $M$ is unbounded. We modify the above 
construction of $u$. Thus, instead of a finite covering of $M$ by balls $B_{i}$, we now use a countable (but locally finite) covering. Further, the radii of the $B_{i}$ may decrease to 0 as $i \rightarrow \infty$. However, there is still a neighborhood $W$ of $M$ such that $L u(x)<0$ if $x \in W \backslash M, u(x) \rightarrow \infty$ if $x \in W \backslash M, d(x, M) \rightarrow 0$; the last relation holds uniformly in $x$ in bounded subsets. The "thickness" of $W \backslash M$ may go to zero at $\infty$.

Now, if the assertion (1.3) is false, then there is an event $G$ with $P_{x}(G)>0$ such that, if $\omega \in G, \xi(t, \omega) \in M$ for some $t=t_{\omega}<\infty$. Introduce the balls $B_{m}=$ $\{y ;|y|<m\}, m$ a positive integer, and the events

$$
G_{m}=\left\{\omega \in G ; \xi(t, \omega) \in B_{m} \text { if } 0 \leq t \leq t_{\omega}\right\}
$$

Clearly $G=\bigcup_{m=1}^{\infty} G_{m}$. Hence there is an $m$ for which $P_{x}\left(G_{m}\right)>0$. But this contradicts Lemma 2.1 in the case where $K=M \cap \bar{B}_{m}, \Omega=B_{m}$.

Remark. Let $M$ be a manifold with boundary. Suppose that $d(x) \geq 3$ if $x \in \partial M$ and $d(x) \geq 2$ and (when $n-k \geq 3)\left(\mathrm{N}_{x}\right)$ holds for each $x \in M$. Then $M$ is nonattainable. Indeed, if $x^{0} \in \partial M$ then we can construct a function satisfying (4.12)(4.14) by the proof of Theorem 3.1. If $x^{0} \in M \backslash \partial M$, then we can construct $u$ satisfying (4.12)-(4.14) as in the proof of Theorem 4.2. Now use partition of unity (as in the proof of Theorem 4.2) in order to complete the proof.

5. $M$ consists of one point and $d=1$. We shall consider primarily the case where $M$ consists of one point $x^{0}$, and $x^{0}=0$. We begin, for simplicity, with the case $n=2$. Without loss of generality we may take $a_{11}(0,0)>0, a_{22}(0,0)=0$. Since $a_{22}(x, y) \geq 0$, we conclude that $\partial a_{22} / \partial x=0, \partial a_{22} / \partial y=0$ at the origin. Hence, if $a_{22}(x, y)$ is in $C^{2}$ in a neighborhood of the origin, $a_{22}(x, y)=O\left(r^{2}\right)$ where $r^{2}=x^{2}+y^{2}$. From the inequality $\left|a_{12}\right| \leq \sqrt{a_{11}} \sqrt{a_{22}}$ we see that $a_{12}(0,0)=0$. Hence, if $a_{12}(x, y)$ is continuously differentiable and $a_{22}(x, x)$ is twice continuously differentiable in a neighborhood of the origin, then

$$
a(x, y)=\left(\begin{array}{cc}
A+o(1) & M x+N y+o(r) \\
M x+N y+o(r) & B x^{2}+C x y+D y^{2}+o\left(r^{2}\right)
\end{array}\right), \quad A>0,
$$

as $r \rightarrow 0$. Since the matrix $a(x, y)$ is positive semidefinite, $B \geq 0, D \geq 0, M^{2} \leq$ $A B, C^{2} \leq 4 B D$.

We shall assume:

$$
B>0 \text {, }
$$

and $|C|,|M|$ are "sufficiently small," so that for some $p>1, q>1, p^{\prime}>1$, $q^{\prime}>1, p_{0}>1, q_{0}>1$, where $1 / p+1 / q=1,1 / p^{\prime}+1 / q^{\prime}=1,1 / p_{0}+1 / q_{0}=1$, and for some $\lambda>0$, the following inequalities hold. 


$$
\begin{aligned}
&|C| \lambda / p+2|M| / p^{\prime}<B \lambda, \\
&|C| / q \leq D, \\
&|M| \lambda / q^{\prime}<2 A, \\
& 4|M| \lambda / q_{0}+2 A<B \lambda, \\
& 4|M| / p_{0}+B \lambda<6 A .
\end{aligned}
$$

Finally, we assume:

$$
\text { If } D=0, \text { then } a_{22}(x, y)=B x^{2}(1+o(1)) .
$$

Notice that if $|M|$ is sufficiently small so that $4|M| / q_{0}<B, 2|M| / p_{0}<3 A$ and

$$
\alpha^{\prime} \equiv \frac{2 A}{B-4|M| / q_{0}}<\frac{2\left(3 A-2|M| / p_{0}\right)}{B} \equiv \alpha^{\prime \prime},
$$

then any $\lambda$ satisfying $\alpha^{\prime}<\lambda<\alpha^{\prime \prime}$.also satisfies (5.6), (5.7).

Regarding the $b_{i}$, we require that $b_{2}(0,0)=0$. Hence, if $b_{2}(x, y)$ is continuously differentiable in a neighborhood of the origin, then

$$
b_{2}(x, y)=c_{1} x+c_{2} y+o(r) \text {. }
$$

Theorem 5.1. Let (5.1)-(5.9) bold. Then, for any $(x, y) \neq(0,0)$,

$$
P_{(x, y)}\{|\xi(t)|=0 \text { for some } t>0\}=0 .
$$

Proof. Let $R(x, y)=x^{4}+\mu x^{2} y^{2}+\lambda y^{2}$ where $\lambda$ is a positive number satisfying (5.3)-(5.7), and $\mu$ is a positive constant to be determined later on. We shall find a function $u=\Phi(R)$ such that, for some small $y>0$,

$$
\begin{gathered}
L \Phi(R) \leq 0 \quad \text { if } 0<R<\gamma, \\
\Phi(R) \rightarrow \infty \quad \text { if } R \rightarrow 0 .
\end{gathered}
$$

By .Lemma 2.1, this will complete the proof of the theorem.

We can write $L u$ in the form $L u=\alpha \Phi^{\prime \prime}(R)+\beta \Phi^{\prime}(R)$. If we show that

$$
\begin{gathered}
\alpha \geq 0, \quad \beta \geq \alpha / R, \\
\Phi^{\prime \prime}(R)+\Phi^{\prime}(R) / R=0, \quad \Phi^{\prime}(R)<0,
\end{gathered}
$$

then (5.11) follows. A solution of (5.14) is given by $\Phi(R)=\log (1 / R)$. With this $\Phi(R),(5.12)$ is also satisfied. Thus, it remains to verify (5.13).

We shall use the following notation: if $E$ is a constant then $\hat{E}$ is a function of the form $E(1+o(1))$. 
Now, by direct calculation one finds that

$$
\begin{aligned}
a= & 16 \hat{A} x^{6}+4 \hat{B} \lambda^{2} x^{2} y^{2}+4 \hat{D} \lambda^{2} y^{4}+4 \hat{C} \lambda^{2} x y^{3}+8 M \lambda x^{4} y, \\
\beta R= & (12 \hat{A}+2 \hat{B} \lambda) x^{6}+\left(12 \hat{A} \lambda+2 \hat{B} \lambda^{2}\right) x^{2} y^{2}+\left(2 D \lambda^{2}+2 \hat{A} \lambda \mu+2 c_{2} \lambda^{2}\right) y^{4} \\
& +2 C \lambda^{2} x y^{3}+2 c_{1} \lambda^{2} x y^{3} .
\end{aligned}
$$

Using the inequalities

$$
\left|x y^{3}\right| \leq x^{2} y^{2} / p+y^{4} / q, \quad\left|x^{4} y\right| \leq x^{2} y^{2} / p^{\prime}+x^{6} / q^{\prime}
$$

and (5.3)-(5.5), we find that $a \geq 0$ (if $D=0$ we use also (5.8)).

In order to show that $\beta R \geq a$, we use the inequalities

$$
\left|x y^{3}\right| \leq \eta x^{2} y^{2}+y^{4} / 4 \eta, \quad\left|x^{4} y\right| \leq x^{2} y^{2} / p_{0}+x^{6} / q_{0},
$$

in both $\alpha$ and $\beta R$. We then obtain the inequality

$$
\beta R-\alpha \geq \hat{\gamma}_{1} x^{6}+\hat{\gamma}_{2} x^{2} y^{2}+\hat{\gamma}_{3} y^{4} \quad\left(\hat{\gamma}_{i}=\gamma_{i}(1+o(1))\right) \text {. }
$$

By (5.6), $\gamma_{1}>0$, and by (5.7), $\gamma_{2}>0$ provided $\eta$ is sufficiently small. Since $\mu$ does not appear in $\gamma_{1}, \gamma_{2}$, and since it appears only in the additive term $2 \hat{A} \lambda \mu$ of $\gamma_{3}$, we can choose $\mu$ so large that $\gamma_{3}>0$. It follows that $\beta R \geq \alpha$. We have thus completed the proof of (5.13).

Remark 1. When $n=1$ and $d\left(x^{0}\right)=1$ then $\sigma\left(x^{0}\right)$ is nondegenerate (i.e., $\sigma\left(x^{0}\right) \neq 0$ ), and (by [6], for instance)

$$
P_{x}\left\{\xi(t)=x^{0} \text { for some } t>0\right\} \rightarrow 1 \text { if } x \rightarrow x^{0} .
$$

Remark 2. The condition (5.2) is essential for the validity of the assertion of Theorem 5.1. Consider, for example, the system

$$
d \xi_{1}=d w_{1}, \quad d \xi_{2}=\sigma\left(\xi_{1}, \xi_{2}\right) d w_{2},
$$

where $\sigma\left(x_{1}, 0\right)=0$. If $\left(\xi_{1}(0), \xi_{2}(0)\right)=(\alpha, 0)$, then the solution is $\xi_{1}(t)=a+$ $w_{1}(t), \xi_{2}(t)=0$. Hence

$$
P_{(a, 0)}\{|\xi(t)|=0 \text { for some } t>0\}=1 .
$$

Remark 3. A quick review of the proof of (5.13) shows that we have actually proved also that $\beta \geq(1+\delta) \alpha / R$ for some sufficiently small $\delta>0$. Hence we can take in the above proof $\Phi(R)=1 / R^{\delta}$.

Consider now the case $n \geq 2$. Without loss of generality we may assume that $a_{11}(0)>0, a_{i i}(0)=0$ if $2 \leq i \leq n$. If $a_{i i}(x)(2 \leq i \leq n)$ is in $C^{2}$ in a neighborhood of 0 then $a_{i i}(x)=O\left(|x|^{2}\right)$. It follows that $a_{1 i}(x)=O(|x|), a_{i j}(x)=O\left(|x|^{2}\right)$ $(2 \leq i, j \leq n)$. 
Setting $y_{j}=x_{j+1}(1 \leq j \leq n-1), m=n-1$, and assuming that the $a_{i j}$ are in $C^{2}$ in a neighborhood of the origin, we then have:

$$
\begin{aligned}
& a_{11}=A+o(1), A>0, \\
& a_{1 j}=M_{j} x_{1}+\sum_{l=1}^{m} M_{j l} y_{l}+o(r) \quad(2 \leq j \leq n),
\end{aligned}
$$

$$
\begin{aligned}
& a_{i j}=B_{j} x_{1}^{2}+\sum_{l=1}^{m} C_{j l} x_{1} y_{l}+\sum_{l, k=1}^{m} D_{j, l k} y_{l} y_{k}+o\left(r^{2}\right) \quad(2 \leq j \leq n), \\
& a_{i j}=E_{i j} x_{1}^{2}+\sum_{l=1}^{m} E_{i j, l} x_{1} y_{l}+\sum_{l, k=1}^{m} E_{i j, l k} y_{l} y_{k}+o\left(r^{2}\right) \quad(2 \leq i, j \leq n) .
\end{aligned}
$$

We shall assume:

$$
\sum_{l, k, i, j}\left(D_{i, l k} \delta_{i j}+E_{i j, l k}\right) y_{l} y_{k} y_{i} y_{j} \geq c|y|^{4} \quad(c>0)
$$

$$
\left|C_{j l}\right|,\left|M_{j}\right|,\left|E_{i j}\right|,\left|E_{i j, k}\right| \text { are sufficiently small. }
$$

Notice that the left-hand side of (5.17) is always $\geq 0$. In case (5.17) does not hold, we shall have to impose further restrictions.

If $c=0$ in (5.17) then $C_{j l}=0, E_{i j, k}=0$ and the terms o(r),o(r $\left.{ }^{2}\right)$ (5.19) occurring in $a_{j j}, a_{i j}$ (in (5.15)) are replaced by $o\left(\left|x_{1}\right|\right)$ and $o\left(x_{1}^{2}\right)$

respectively.

Theorem 5.2. Let (5.15), (5.16) bold. Assume also that either (5.17), (5.18), hold, or (5.19) holds and the $\left|M_{j}\right|,\left|E_{i j}\right|$ are sufficiently small. Then,

$$
P_{x}\{\xi(t)=0 \text { for some } t>0\}=0 \text { if } x \neq 0 \text {. }
$$

The proof is similar to the proof of Theorem 5.1. We now take $u=\Phi(R)$ with $\Phi$ as before, but with

$$
R(x)=x_{1}^{4}+\mu \sum_{j=1}^{m} x_{1}^{2} y_{j}^{2}+\lambda \sum_{j=1}^{m} y_{j}^{2} ;
$$

$\lambda$ is a suitable positive number and $\mu$ is a sufficiently large positive number.

Theorems 5.1, 5.2 can be extended to manifolds $M$ of special form. As a trivial example, take $n=3, M$ the $z \cdot a x$ is, and $a_{i j}$ for $1 \leq i, j \leq 2$ as in Theorem 
5.1. Then $d=1$ along $M$, and $M$ is nonattainable. The proof uses the same function $u=\Phi(R)$ as in the proof of Theorem 5.1.

6. The case $d(x)=0$. The following theorem was proved by Friedman and Pinsky [4].

Theorem 6.1. Let $G$ be a closed bounded domain in $R^{n}$ with $C^{3}$ boundary $M$, and denote by $\nu=\left(\nu_{1}, \cdots, \nu_{n}\right)$ the outward normal to $G$ at $M$. Let (A) bold, and assume that

$$
\begin{gathered}
\sum_{i, j=1}^{n} a_{i j} \nu_{i} \nu_{j}=0 \text { on } M, \\
\langle b, v\rangle+\sum_{i, j=1}^{n} a_{i j} \frac{\partial^{2} \rho}{\partial x_{i} \partial x_{j}} \geq 0 \text { on } M,
\end{gathered}
$$

where $\rho(x)=\operatorname{dist}(x, M)$ if $x \notin \operatorname{int} G$. Then, for any $x \notin G$,

$$
P_{x}\{\xi(t) \in M \text { for some } t>0\}=0 \text {. }
$$

If the inequality in (6.2) is reversed at one point $x_{0}$ of $M$, then the assertion (6.3) is false; in fact (cf. [4]),

$$
P_{x}\{\xi(t) \in M \text { for some } t>0\} \rightarrow 1 \text { if } x \notin G, x \rightarrow x^{0} .
$$

Notice that the condition (6.1) means that $d(x)=0$ along $M$.

We also note (see [4]) that, when the $a_{i j}$ belong to $C^{1}$ in a neighborhood of $M$, the condition (6.2) is equivalent to:

$$
\sum_{i=1}^{n}\left(b_{i}-\sum_{j=1}^{n} \frac{\partial a_{i j}}{\partial x_{j}}\right) \nu_{i} \geq 0 \text { on } M .
$$

The proof of Theorem 6.1 follows by producing a function $u$ satisfying $L u \leq$ $\mu u$ in a $\hat{G}$-neighborhood of $M, \hat{G}=R^{n} \backslash G, \mu>0, u(x) \rightarrow \infty$ if $x \in \hat{G}, \rho(x) \rightarrow 0$. Such a function is

$$
u(x)=1 /(\rho(x))^{\epsilon} \text { for any } \epsilon>0 .
$$

Suppose now that $G$ is a bounded, closed and convex domain, with piecewise $C^{3}$ boundary. Thus each point $x$ of the boundary $M$ lies on a finite number of $C^{3}$ $(n-1)$-dimensional submanifolds of $M$, say $M_{i_{1}}, \cdots, M_{i_{s}}$. The ir intersection is a $k$-dimensional $C^{3}$ manifold through $x(k=n-s)$. Denote by $N_{x}$ the $(n-k)$ dimensional space of the normals to this submanifold at $x$.

The function $D_{x} \rho(x)$ is continuous. On the other hand, $D_{x}^{2} \rho(x)$ is piecew ise continuous; denote by $\Sigma$ the set of its discontinuities. 
Theorem 6.1 extends to the present case provided (6.1) holds for any $x \in M$, $\nu \in N_{x}$, and provided (6.2) is replaced by

$$
\varliminf_{y \rightarrow x} \frac{1}{\rho(y)}\left[\sum_{i=1}^{n} b_{i}(y) \frac{\partial}{\partial y_{i}} \rho(y)+\sum_{i, j=1}^{n} a_{i j}(y) \frac{\partial^{2}}{\partial y_{i} \partial y_{j}} \rho(y)\right] \geq-C
$$

$(y \notin G \cup \Sigma, C$ positive constant).

Notice that condition (6.1) for all $\nu \in N_{x}$ can be interpreted as $d_{M^{\perp}}(x)=0$, when the notion of $d_{M^{\perp}}$ is extended in a natural way to the case of a piecewise smooth manifold.

When $\operatorname{dim} N_{x}=n$, the conditions (6.1) for all $\nu \in N_{x}$ and (6.6) reduce to $\sigma(x)=0, b(x)=0$.

Suppose next that $M$ is a piecewise $C^{3}$ bounded submanifold in $R^{n}$, of any dimension $k(1 \leq k \leq n-1)$, with piecewise $C^{3}$ boundary $\partial M$. We can still extend Theorem 6.1 (taking $u(x)=1 /(d(x, M))^{\epsilon}, \epsilon>0$ ) provided the following conditions hold:

(i) $d(x, M)$ is continuously differentiable and its second derivative is piecewise continuous, in some $\hat{M}$-neighborhood of $M ; \hat{M}=R^{n} \backslash M$; denote by $\hat{\Sigma}$ the set of discontinuities of $D_{x}^{2} d(x, M)$ in $\hat{M}$.

(ii) For any $x \in$ int $M,(6.1)$ holds for all $\nu \in N_{x}$ ( $N_{x}$ is the space of normals to $M$ at $x$ ), and

$$
\varliminf_{y \rightarrow x} \frac{1}{d(y, M)}\left[\sum_{i=1}^{n} b_{i}(y) \frac{\partial}{\partial y_{i}} d(y, M)+\sum_{i, j=1}^{n} a_{i j}(y) \frac{\partial^{2}}{\partial y_{i} \partial y_{j}} d(y, M)\right] \geq-C
$$

( $y \notin M \cup \hat{\Sigma}, C$ positive constant) uniformly with respect to $x$.

(iii) For any $x \in \partial M,(6.1)$ holds for all $\nu$ normal to $\partial M$ at $x$, and (6.7) holds.

Remark 1. In [4], Theorem 6.1 was extended to $G$ convex with piecewise $C^{3}$ boundary under the assumption that $a_{i j} \in C^{2}$. Using Lemma 2.1, we see that this assumption is not needed.

Remark 2. In the proof of Lemma 1.1, we had to use Itô's formula for a $C^{1}$ function $u$ with piecewise continuous second derivatives, satisfying $L u \leq \mu u$. We extended $u$ into $U$ and mollified $U$ into $U_{\lambda}$; then applied Itô's formula to $U_{\lambda}$, and finally let $\lambda \rightarrow 0$. The same procedure can be carried out in the proof of Theorem 2.2 in [4]. This simplifies that proof and also enables us to eliminate the restriction $(D)$ (made in that theorem).

7. Mixed case: an application to the Dirichlet problem. Set $d(x)=r_{M^{\perp}}(x)$, $d^{\prime}(x)=r_{(\partial M)^{\perp}}^{\perp}(x)$. We shall consider the case where $n=2, M$ is an arc, and

$$
d(x)=0 \quad \text { if } x \in M, \quad d(x)=1 \quad \text { if } x \in \partial M .
$$


One can also consider, by the same method, other mixed cases. The motivation for studying the particular case (7.1) arises from an application to the Dirichlet problem; this will be considered later on.

The idea for handling the mixed case $(7.1)$ is to form two functions, $u_{1}$ and $u_{2}$, such that:

(i) $u_{1}$ is a function constructed for the case $d(x)=0$ (in $\$ 6$ );

(ii) $u_{2}$ is a function constructed for the case $d^{\prime}(x)=1$ (in $\$ 5$ );

(iii) $u_{1}$ and $u_{2}$ fit together in a continuously differentiable manner.

For simplicity we shall deal primarily with the case:

$$
M=\left\{\left(x_{1}, x_{2}\right) ; x_{1}=0,0 \leq x_{2} \leq \beta\right\} .
$$

The case of a general arc $M$ follows by first performing a local diffeomorphism, mapping the arc onto a linear segment as in (7.2).

Let $\Omega$ be a bounded closed domain lying in the half-plane $x_{1} \geq 0$, with boundary $\partial_{1} \Omega \cup \partial_{2} \Omega$, where $\partial_{1} \Omega=\left\{\left(x_{1}, x_{2}\right) ;-a \leq x_{1} \leq a, x_{2}=0\right\}$ and $\partial_{2} \Omega$ lies in the half-plane $x_{2}>0$. We assume that $M \subset \Omega$.

The stochastic differential system is

$$
d \xi_{i}=\sum_{s=1}^{2} \sigma_{i s}(\xi) d w_{s}+b_{i}(\xi) d t \quad(i=1,2) .
$$

Denote by $\tau$ the exit time from $\Omega$. In view of the application for the Dirichlet problem, we are interested in the process $\xi(t)$ only as long as $t<\tau$. Thus, we would like to prove that $M$ is nonattainable in time $<\tau$, i.e.,

$$
P_{x}\{\xi(t) \in M \text { for some } t<\tau\}=0 \text { if } x \in \Omega \backslash M \text {. }
$$

First we assume that $(6.1),(6.4)$ hold with respect to both sides of $M$, i.e., if $a=\sigma \sigma^{*} / 2$ then

$$
\begin{gathered}
a_{11}\left(0, x_{2}\right)=0 \text { for } 0 \leq x_{2} \leq \beta \\
b_{1}\left(0, x_{2}\right)-\frac{\partial a_{11}\left(0, x_{2}\right)}{\partial x_{1}}-\frac{\partial a_{12}\left(0, x_{2}\right)}{\partial x_{2}}=0 \text { if } 0 \leq x_{2} \leq \beta .
\end{gathered}
$$

If the point $(0, \beta)$ lies on the boundary of $\Omega$, then (7.4) follows from the proof of Theorem 6.1 (when slightly modified). Recall that we apply here Lemma 2.1 with any function

$$
u(x)=c /\left(x_{1}^{2}\right)^{\epsilon} \quad(c>0, \epsilon>0) .
$$

In applications, however, $\beta$ may be small, so that

$$
(0, \beta) \epsilon \text { int } \Omega \text {. }
$$


We shall henceforth assume that (7.8) holds, and that not all the $a_{i j}(0, \beta)$ $(1 \leq i, j \leq 2)$ vanish. (If they all vanish then (7.4) again follows from the results of $\$ 6$.

Assuming the $a_{i j}$ to be in $C^{2}$ in a neighborhood of $(0, \beta)$, and recalling (7.5), we then have:

$$
a\left(x_{1}, x_{2}\right)=\left(\begin{array}{cc}
B x_{1}^{2}+C x_{1}\left(x_{2}-\beta\right)+D\left(x_{2}-\beta\right)^{2}+o\left(r^{2}\right) & M x_{1}+N\left(x_{2}-\beta\right)+o(r) \\
M x_{1}+N\left(x_{2}-\beta\right)+o(r) & A+o(1)
\end{array}\right) \text { 9) }
$$

$$
A>0,
$$

where $r^{2}=x_{1}^{2}+\left(x_{2}-\beta\right)^{2}$. We shall require (cf. (5.9)) that

$$
b_{1}\left(x_{1}, x_{2}\right)=c_{1} x_{1}+c_{2}\left(x_{2}-\beta\right)+o(r) .
$$

From (7.10), (7.6) it follows that $N=0$ in (7.9). We finally require that either

$$
D>0, B>0,|C| \text { is sufficiently small, }
$$

or

$$
D>0, B=0, C=0 \text {, and } a_{11}\left(x_{1}, x_{2}\right)=B x_{1}^{2}(1+o(1)) .
$$

Consider the function

$$
u(x)=1 /(R(x))^{\delta} \quad(\delta>0),
$$

where $R(x)=\left(x_{2}-\beta\right)^{4}+\mu\left(x_{2}-\beta\right)^{2} x_{1}^{2}+\lambda x_{1}^{2}$. By Remark 3 at the end of the proof of Theorem 5.1, Lu $\leq 0$ if $0<x_{1}^{2}+\left(x_{2}-\beta\right)^{2}<\epsilon_{0}$ for some $\epsilon_{0}>0$, provided $\delta$ is sufficiently small; here $\mu, \lambda$ are suitable positive constants.

Note that the function

$$
d(x)= \begin{cases}R(x) & \text { if } x_{2}>\beta \\ \lambda x_{1}^{2} & \text { if } x_{2}<\beta\end{cases}
$$

is $C^{1}$ and piecewise $C^{2}$. Recalling (7.7), (7.13), we conclude that the function $u(x)=1 /(d(x))^{\delta}$ is $C^{1}$ and piecewise $C^{2}$ in $\Omega \backslash M$, and $L u \leq 0$ for $x$ in $(\Omega \backslash M)$ neighborhood of $M, x_{2} \neq \beta, u(x) \rightarrow \infty$ if $x \in \Omega \backslash M, d(x, M) \rightarrow 0$. Hence, by Lemma 2.1, (7.4) holds. We sum up:

Theorem 7.1. Let (7.5), (7.6), (7.9), (7.10) bold, and let (7.11) or (7.12) bold. Then (7.4) is satisfied.

An application. In [5] Friedman and Pinsky have considered the Dirichlet problem for $n=2$, 


$$
\begin{aligned}
L u & =0 \text { in a bounded domain } G, \\
u & =f \text { on } \Sigma_{2} \cup \Sigma_{3}, \\
u(x) & \rightarrow f_{i}^{+} \text {if } x \rightarrow p_{i}, \quad x \in N_{i}^{+} \quad(1 \leq i \leq l), \\
u(x) & \rightarrow f_{i}^{-} \text {if } x \rightarrow p_{i}, \quad x \in N_{i}^{-} \quad(1 \leq i \leq l) ;
\end{aligned}
$$

$f$ is a given continuous function, and the $f_{i}^{+}, f_{i}^{-}$are given numbers. Here $\Sigma_{3}$ is the part of the boundary where $\Sigma a_{i j} \nu_{i} \nu_{j}>0 ; \Sigma_{2}$ is the part where (6.1) holds and

$$
\sum_{i=1}^{2}\left(b_{i}-\sum_{j=1}^{2} \frac{\partial a_{i j}}{\partial x_{i}}\right) \nu_{i}>0 \quad(\nu \text { outward normal })
$$

and $\Sigma_{3}$ is the remaining boundary. The points $p_{i}$ lie in $\Sigma_{1}, N_{i}^{+} \cap N_{i}^{-}=\varnothing$, and the closure of $N_{i}^{+} \cup N_{i}^{-}$constitutes a $\bar{G}$-neighborhood of $p_{i}$. The open sets $N_{i}^{+}$, $N_{i}^{-}$have a common boundary $\hat{\Delta}_{i}$. The location of the points $p_{i}$ can be determined explicitly from the $a_{i j}, b_{i}$. One of the assumptions made in [5] is that there is a curve $\Delta_{i}$, initiating at $p_{i}$ and terminating on the boundary of $G$, such that $\Delta_{i}$ is an extension of $\hat{\Delta}_{i}$ and such that the conditions (6.1), (6.2) (with $n=2$ ) hold along $\Delta_{i}$, from both sides of it. The curve $\Delta_{i}$ is called a "boundary spoke". The rank of $a(x)$ for any $x \in \Delta_{i} \cap G$ is 1 .

The existence of such a curve $\Delta_{i}$ was assumed in order to assert that $\xi(t)$ does not cross $\hat{\Delta}_{i}$ if $t$ is sufficiently large, and $t<\tau$.

Using Theorem 7.1, we can now replace the assumption regarding the "boundary spoke" $\Delta_{i}$ by the following set of assumptions:

(a) There is a curve $\tilde{\Delta}_{i}$ initiating at $p_{i}$, containing $\hat{\Delta}_{i}$ and lying in $G$ (except for its initial point $p_{i}$ ); denote its end point by $q_{i}{ }^{\bullet}$

(b) The conditions $(6.1),(6.2)$ (with $n=2$ ) hold along $\tilde{\Delta}_{i}$, from both sides of it.

(c) The conditions "analogous" to (7.9), (7.10) and either (7.11) or (7.12) hold.

By the "analogous" conditions we mean the following:

Perform a local diffeomorphism in a neighborhood $W$ of $\tilde{\Delta}_{i}$ which maps $\tilde{\Delta}_{i}$ onto the line segment (7.2) and $W \cap \partial G$ onto a segment $\left\{\left(x_{1}, 0\right) ;-\alpha<x_{1}<\alpha\right\}$. Then, the transformed $a_{i j}, b_{i}$ satisfy (7.9), (7.10) and either (7.11) or (7.12).

We sum up:

The "long" "boundary spoke" $\Delta_{i}$ going from $p_{i}$ to the boundary of $G$ can be replaced by a "short" "boundary spoke" $\tilde{\Delta}_{i}$ going from $p_{i}$ to a point $q_{i}$ in $G$, provided the condition (c) is satisfied at $q_{i}$. 


\section{REFERENCES}

1. A. Bonami, N. Karoui, B. Roynette and H. Reinhard, Processus de diffusion associé à un opérateur elliptique dégénéré, Ann. Inst. H. Poincaré Sect. B (N. S.) 7 (1971), 31-80. MR $44 \# 7637$.

2. A. Friedman, Partial differential equations, Holt, Rinehart and Winston, New York, 1969.

3. A. Friedman and M. A. Pinsky, Asymptotic behavior of solutions of linear stochastic differential systems, Trans. Amer. Math. Soc. 181 (1973), 1-22.

4. - Asymptotic stability and spiraling properties for solutions of stochastic equations, Trans. Amer. Math. Soc. 186 (1973), 331-358.

5. - Dirichlet problem for degenerate elliptic equations, Trans. Amer. Math. Soc. 186 (1973), 359-383.

6. I. I. Gihman and A. V. Skorohod, Stochastic differential equations, Naukova Dumka, Kiev, 1968. (Russian). MR 41 \#7777.

DEPAR TMENT OF MATHEMATICS, NORTHWESTERN UNIVERSITY, EVANSTON, ILLINOIS 60201 